\title{
MSVAT-SPACE-STIR and SEMAC-STIR for Reduction of Metallic Artifacts in 3T Head and Neck MRI
}

\author{
(D)T. Hilgenfeld, (D) M. Prager, (D) F.S. Schwindling, (D) M. Nittka, (D). Rammelsberg, (DM. Bendszus, (D) S. Heiland, and (D) A. Juerchott
}

\begin{abstract}
BACKGROUND AND PURPOSE: The incidence of metallic dental restorations and implants is increasing, and head and neck MR imaging is becoming challenging regarding artifacts. Our aim was to evaluate whether multiple-slab acquisition with view angle tilting gradient based on a sampling perfection with application-optimized contrasts by using different flip angle evolution (MSVAT-SPACE)-STIR and slice-encoding for metal artifact correction (SEMAC)-STIR are beneficial regarding artifact suppression compared with the SPACE-STIR and TSE-STIR in vitro and in vivo.
\end{abstract}

MATERIALS AND METHODS: At 3T, 3D artifacts of 2 dental implants, supporting different single crowns, were evaluated. Image quality was evaluated quantitatively (normalized signal-to-noise ratio) and qualitatively ( 2 reads by 2 blinded radiologists). Feasibility was tested in vivo in 5 volunteers and 5 patients, respectively.

RESULTS: Maximum achievable resolution and the normalized signal-to-noise ratio of MSVAT-SPACE-STIR were higher compared with SEMAC-STIR. Performance in terms of artifact correction was dependent on the material composition. For highly paramagnetic materials, SEMAC-STIR was superior to MSVAT-SPACE-STIR (27.8\% smaller artifact volume) and TSE-STIR ( $93.2 \%$ less slice distortion). However, MSVAT-SPACE-STIR reduced the artifact size compared with SPACE-STIR by $71.5 \%$. For low-paramagnetic materials, MSVAT-SPACE-STIR performed as well as SEMAC-STIR. Furthermore, MSVAT-SPACE-STIR decreased artifact volume by $69.5 \%$ compared with SPACE-STIR. The image quality of all sequences did not differ systematically. In vivo results were comparable with in vitro results.

CONCLUSIONS: Regarding susceptibility artifacts and acquisition time, MSVAT-SPACE-STIR might be advantageous over SPACE-STIR for high-resolution and isotropic head and neck imaging. Only for materials with high-susceptibility differences to soft tissue, the use of SEMAC-STIR might be beneficial. Within limited acquisition times, SEMAC-STIR cannot exploit its full advantage over TSE-STIR regarding artifact suppression.

ABBREVIATIONS: CCT-T = porcelain-fused-to-metal nonprecious alloy crown with titanium implant; $M A V R I C=$ multiacquisition with variable resonance image combination; MSVAT-SPACE = multiple-slab acquisition with view angle tilting gradient based on SPACE; nSNR = normalized SNR; SEMAC = slice-encoding for metal artifact correction; SPACE = sampling perfection with application-optimized contrasts by using different flip angle evolutions; Z-T = monolithic zirconia crown with titanium implant

M R imaging has become a widely used technique for the head and neck area. Image quality, however, it is often impaired by metallic dental restorations and implant-supported prostheses. ${ }^{1} \mathrm{MR}$ image quality is affected by dental metals spoiling the

Received October 15, 2017; accepted after revision March 30, 2018.

From the Department of Neuroradiology, (T.H., M.P., M.B., S.H., A.J.) and Section of Experimental Radiology (M.P., S.H.), University of Heidelberg, Heidelberg, Germany; Department of Prosthodontics (F.S.S., P.R.), Heidelberg University Hospital, Heidelberg, Germany; and Siemens Healthcare (M.N.), Erlangen, Germany. Tim Hilgenfeld and Marcel Prager contributed equally to this work. The study was supported, in part, by Dietmar Hopp Stiftung (project no. 23011228). Please address correspondence to Alexander Juerchott, MD, Department for Neuroradiology, University Hospital, Im Neuenheimer Feld 400, 69120 Heidelberg, Germany; e-mail: Alexander.Juerchott@med.uni-heidelberg.de

http://dx.doi.org/10.3174/ajnr.A5678 homogeneity of the static magnetic field $\left(\mathrm{B}_{0}\right)^{2,3}$ and by eddy currents in response to alternating gradients and radiofrequency magnetic fields. ${ }^{4,5}$ In the elderly, besides dental restoration materials, metallic implants and their crowns are a major source of artifacts. In Germany for instance, the prevalence of dental implants has increased 10-fold compared with $1997,{ }^{6}$ which is caused by an increased patient life expectancy and a broadening of implant indications. Therefore, artifact reduction has become increasingly important in head and neck imaging.

To address the decreased image quality due to metallic implants, several sequences for metal artifact reduction were developed such as view angle tilting, slice-encoding for metal artifact correction (SEMAC), multiacquisition with variable resonance image combination (MAVRIC; GE Healthcare, Milwaukee, Wisconsin), the 


\begin{tabular}{|c|c|c|c|c|c|c|c|c|c|}
\hline Sequence & $\mathrm{TR} / \mathrm{TE}$ (ms) & $\begin{array}{c}\text { Voxel } \\
\text { Size }(\mathrm{mm})\end{array}$ & FOV (mm) & Matrix & $\begin{array}{l}\text { Readout } \\
\text { Bandwidth } \\
(\mathrm{Hz} / \mathrm{Px})\end{array}$ & Slices & $\begin{array}{l}\text { Slice-Encoding } \\
\text { Steps or } \\
\text { Oversampling (\%) }\end{array}$ & VAT & $\begin{array}{c}\text { Time } \\
\text { (min:sec) }\end{array}$ \\
\hline SPACE-STIR & $2500 / 131$ & $0.55 \times 0.55 \times 0.55$ & $140 \times 124$ & 256 & 501 & 72 & 55.6 & No & 14:02 \\
\hline MSVAT-SPACE-STIR & $2500 / 199$ & $0.55 \times 0.55 \times 0.55$ & $140 \times 84$ & 256 & 528 & 72 & 55.6 & Yes & $06: 04$ \\
\hline TSE-STIR & $5100 / 44$ & $0.59 \times 0.59 \times 1.5$ & $150 \times 150$ & 256 & 592 & 25 & No & No & $03: 36$ \\
\hline SEMAC-STIR & $5100 / 45$ & $0.59 \times 0.59 \times 1.5$ & $150 \times 150$ & 256 & 592 & 25 & 4 & Yes & 06:19 \\
\hline
\end{tabular}

Note:-VAT indicates view angle tilting.

multiple-slab acquisition with view angle tilting gradient based on a sampling perfection with application-optimized contrasts by using different flip angle evolutions (SPACE) sequence (MSVAT-SPACE; Siemens, Erlangen, Germany), and combinations of these techniques such as MAVRIC-SEMAC. ${ }^{7-12}$ These new techniques for artifact reduction were predominantly developed and tested for orthopedic and neurosurgical applications. ${ }^{13,14}$ Until now, little attention has been paid to the head and neck area. Moreover, results of previous studies are of limited transferability because the amount of material, shape, and materials is different in the head and neck area compared with orthopedic or neurosurgical implants, and all these characteristics influence artifact size.

The combination of these pulse sequences with STIR-based fat suppression, as the most reliable fat-suppression technique in the presence of $\mathrm{B}_{0}$ inhomogeneities, ${ }^{15}$ might be beneficial in cases with metallic dental materials and suspected osteomyelitis (evaluation of bone marrow edema), head and neck tumors (defining tumor margins), or injury of the inferior alveolar nerve (evaluation of nerve signal intensity). ${ }^{16}$ Furthermore, previous studies focused on the maximum achievable reduction of artifacts and an ideal comparison of pulse techniques (identical imaging parameters) at the expense of long acquisition times. This focus severely limits the implementation in routine protocols, especially because the head and neck area is extremely sensitive to motion artifacts due to breathing and swallowing. Therefore, we aimed to investigate how well these techniques perform within a limited acquisition time in the presence of commercially available dental implants with supported prostheses compared with standard sequences. To reach this goal, the main tasks were the following:

1) Quantify and compare metal-induced artifact volumes (signal loss and pileup) for 2 commercially available implant-supported prostheses using anisotropic (TSE-STIR, SEMAC-STIR) and isotropic (SPACE-STIR, MSVAT-SPACE-STIR) sequences

2) Compare image quality of all sequences qualitatively and quantitatively

3) Test the feasibility of all sequences in 5 volunteers with metallic dental materials and the sequence providing the best trade-off among artifact reduction, resolution, and image quality in 5 patients.

\section{MATERIALS AND METHODS MR Imaging and Sequences}

A 3T MR imaging system (Magnetom Trio, a Tim system; Siemens), a 16-channel multipurpose surface coil (Variety; NORAS MRI Products; Höchberg, Germany) for the in vitro experiments, and a 15-channel surface coil (Mandibula; NORAS MRI Products) for the in vivo measurements were used.
The SEMAC prototype sequence applies additional phaseencoding steps in the slice direction to correct for distortions of the excited slice profile ${ }^{17}$ and also incorporates view angle tilting to correct for in-plane distortions. ${ }^{11}$ The MSVATSPACE prototype sequence uses slab-selective excitation and refocusing radiofrequency pulses that allow interleaved multislab acquisitions. ${ }^{18}$

Because we aimed for both short acquisition times that allow clinical application and maximum artifact reduction of each specific sequence, individual sequence optimization ended with differences in sequence parameters but an identical, relatively short acquisition time. First, MSVAT-SPACE-STIR and SEMAC-STIR were optimized for artifact reduction in the presence of implants (eg, by changing the voxel size, readout bandwidth, slice oversampling, and number of slice-encoding steps). Second, the SNR was optimized for these 2 sequences (eg, by changing the turbo factor or number of averages). Third, standard sequences (SPACESTIR, TSE-STIR) with imaging parameters as similar as possible to MSVAT-SPACE-STIR and SEMAC-STIR were implemented for comparison. Spectral coverage for off-resonance frequencies was slightly lower for MSVAT-SPACE-STIR $( \pm 2 \mathrm{kHz})$ than for SEMAC-STIR $( \pm 2.8 \mathrm{kHz})$. Due to restrictions in the prototype sequences, the bandwidths of inversion and excitation could not be perfectly matched (MSVAT-SPACE-STIR, $1 / 1.4 \mathrm{kHz}$; SEMAC-STIR, 1.72/1.4 kHz). For a reduction in scanning time, a generalized autocalibrating partially parallel acquisition was used in all sequences, and partial Fourier, for SPACE-STIR and MSVAT-SPACE-STIR. For SEMAC-STIR and TSE-STIR, a flip angle of $150^{\circ}$ was used. Relevant parameters of all sequences are shown in the Table.

\section{Evaluation of Artifact Volume}

Besides the sizes and shape of materials, their magnetic susceptibility heavily influences the artifact volume. Schenck ${ }^{2}$ classified 3 groups of materials because of their differences in magnetic susceptibility. To cover the worst- and best-case scenarios in a realistic setting, we used 2 commercially available implant-supported single crowns for the evaluation of artifact suppression. The single crowns of both prostheses were made of porcelain-fused-to-metal nonprecious alloy (CCT-T) and monolithic zirconia (Z-T), respectively. The CCT-T crown consisted of Cobalt (61\%), Chrome (28\%), and Tungsten (11\%) and belongs to group 1, predicting large artifacts. In contrast, the crown of the Z-T sample consisted of Zirconia (92\%) and belongs to group 3, resulting in no or only minimal artifacts. The implant body, abutment, and abutment screw of both implants were made of Titanium (diameter $\times$ length: $4.3 \times 10 \mathrm{~mm}$ [CCT-T], $4.3 \times 13 \mathrm{~mm}$ [Z-T]; NobelReplace; Nobel Biocare, Zurich, Switzerland). 
Both samples were embedded in a mixture of semisynthetic fat (58.8\%), water (40\%), and macrogol-8-stearate (1.2\%). Artifact volume (signal loss and pileup artifacts) was determined by a semiautomatic threshold-based process with AMIRA 3D software (FEI, Hillsboro, Oregon) as described before. ${ }^{19}$ Quantification of pileup artifacts was performed to serve as an indicator for slice distortions. In vitro results were compared with measured artifact areas in vivo in 5 volunteers with metallic dental materials. For each volunteer, the maximum artifact area was determined in 1 slice 3 times by 2 readers (reader 1 twice, reader 2 once). The results were averaged.

\section{Qualitative Image Review}

For analysis of image quality and to test the sequences in a realistic setting, we performed ex vivo measurements in 2 fresh porcine heads with inserted implant samples. For preparation of the implant site in the anterior section of the mandible, a pilot drill, 1.5 $\mathrm{mm}$ in diameter, and spiral drills, 2.8 and $3.5 \mathrm{~mm}$ in diameter, were used. Both implants were tested consecutively in both porcine heads. Positioning of the longitudinal axis of the implants, porcine teeth, and surface coil was like that in the in vivo situation. Two radiologists (both with 4 years' experience in head and neck imaging) independently assessed all images of the 2 porcine heads twice, with an interval of 2 months to exclude learning bias. Both readers were blinded to the type of sequence. Both observers were asked to identify 8 different anatomic structures of the mandible (cortical bone, trabecular bone, lamina dura, tooth root, pulp chamber, apical foramen, periodontal space, and enamel/dentin). A 5-point scale was used to assess the visibility of anatomic structures as described before. ${ }^{8}$ For visualization of anatomic structures, grade 5 indicated that the anatomic structure was not visible; grade 4 , that $<25 \%$ of the anatomic structure was visible; grade 3 , visualization of $25 \%-50 \%$; grade 2 , visualization of $50 \%-75 \%$; and grade 1 , visualization of $>75 \%$.

Evaluation of motion artifacts in 5 patients was performed at 5 anatomic positions (lymph nodes in lymph node level II, submandibular gland, maxillary artery, inferior alveolar nerve, and masseter muscle). Evaluation of motion artifacts was performed as well on a 5-point scale: 5 , anatomic structure not visible; 4 , severe artifacts but contours could be delineated; 3 , moderate artifacts that allow partial visibility of internal structures; 2 , minor artifacts with good delineation of internal structures; and 1, no artifacts at all.

\section{Quantitative Image Review}

A phantom with 4 tubes (CRYO.S; Greiner Bio-One, Frickenhausen, Germany) containing water was used for normalized SNR (nSNR) measurements. Because a phased array radiofrequency coil was used, we determined the SNR by calculating the dynamic noise and the signal within the same ROI from 25 repetitions of each sequence. ${ }^{20}$ Due to the long acquisition time of the conventional SPACE-STIR and the need for several repetitions of each sequence, evaluation of SNR in vivo was not possible. ROIs of $10 \mathrm{~mm}$ in diameter were placed manually in each of the 4 tubes in 1 slice. A Matlab script (MathWorks, Natick, Massachusetts) allowed copying the ROIs to the same position on MR images of all sequences and repetitions. Due to the long acquisition time, signal drift had to be considered. ${ }^{21}$ Because signal drift was linear in all our measurements, we used linear regression to exclude the bias effects of signal drift. Finally, for a better comparability of SNR among different sequences, the SNR was normalized to voxel size and measurement time (nSNR, formula 1):

$$
n S N R=\frac{\text { mean }(S N R)}{\sqrt{T} \times V} .
$$

Formula 1: Calculation of normalized SNR; mean (SNR), calculated $S N R$ within the ROI; $T$, acquisition time in seconds; $V$, voxel volume in cubic millimeters.

\section{Patient and Volunteer Recruitment}

This observational, prospective study was approved by the institutional ethics committee (approval number S-452/2010; University of Heidelberg), and written informed consent was obtained from all participants. Artifact size of all sequences was evaluated in 5 volunteers with metallic dental materials disturbing the $\mathrm{B}_{0}$. Furthermore, 1 sequence was tested in 5 patients presenting with various head and neck diseases (osteomyelitis, injury of the inferior alveolar nerve, jaw tumor, and drained abscess in the mandible), and motion artifacts were analyzed.

\section{Statistical Analysis}

Due to multiple comparisons, a 2-way analysis of variance with pair-wise post hoc Tukey tests was used for comparison of in vitro artifact volumes using SPSS 22 (IBM, Armonk, New York). Multiple comparisons of nSNR and in vivo artifact areas among all sequences for each volunteer were performed using a 1-way ANOVA with post hoc Tukey tests. Categoric data (visibility scores of image quality) were analyzed with the Fisher exact test after dichotomization of the scores into 2 groups ("good visibility," scores 1-2 and "unsatisfactory visibility," scores 3-5). The Cohen $\kappa$ statistic ( $\kappa$-value) was calculated to determine the interand intrarater agreement of image quality and interpreted as published before. ${ }^{22}$

\section{RESULTS}

\section{In Vitro Analysis}

Quantification of artifact volume revealed only minor intrarater variability (mean, $1.6 \%$; minimum, $0.1 \%$; maximum, $5.5 \%$ ) and minor interrater variability (mean, $1.1 \%$; minimum, $0.1 \%$; maximum, 2.8\%).

Overall, artifact size of the CCT-T sample was significantly larger compared with the Z-T sample in all tested sequences $(P<.001)$. The artifact volume of CCT-T was between $9.8 \pm 1.4$-fold (SEMACSTIR, 2.7 versus $0.3 \mathrm{~mL}$ ) and $26.4 \pm 6.6$-fold (SPACE-STIR, 12.9 versus $0.4 \mathrm{~mL}$ ) larger than the artifact volume of Z-T (Fig 1).

\section{Impact of Sequence Type on Artifact Volume}

Comparing all STIR sequences with each other, we found a significant decrease of $71.5 \% \pm 0.1 \%$ in artifact volume in MSVATSPACE-STIR compared with SPACE-STIR for the CCT-T sample $(P<.001)$ and $69.7 \% \pm 5.4 \%$ for the $\mathrm{Z}$-T sample $(P<$ .001 , Figs 1 and 2$)$. As a result, in vivo visibility of anatomic structures in direct proximity to a retainer and a dental filling 

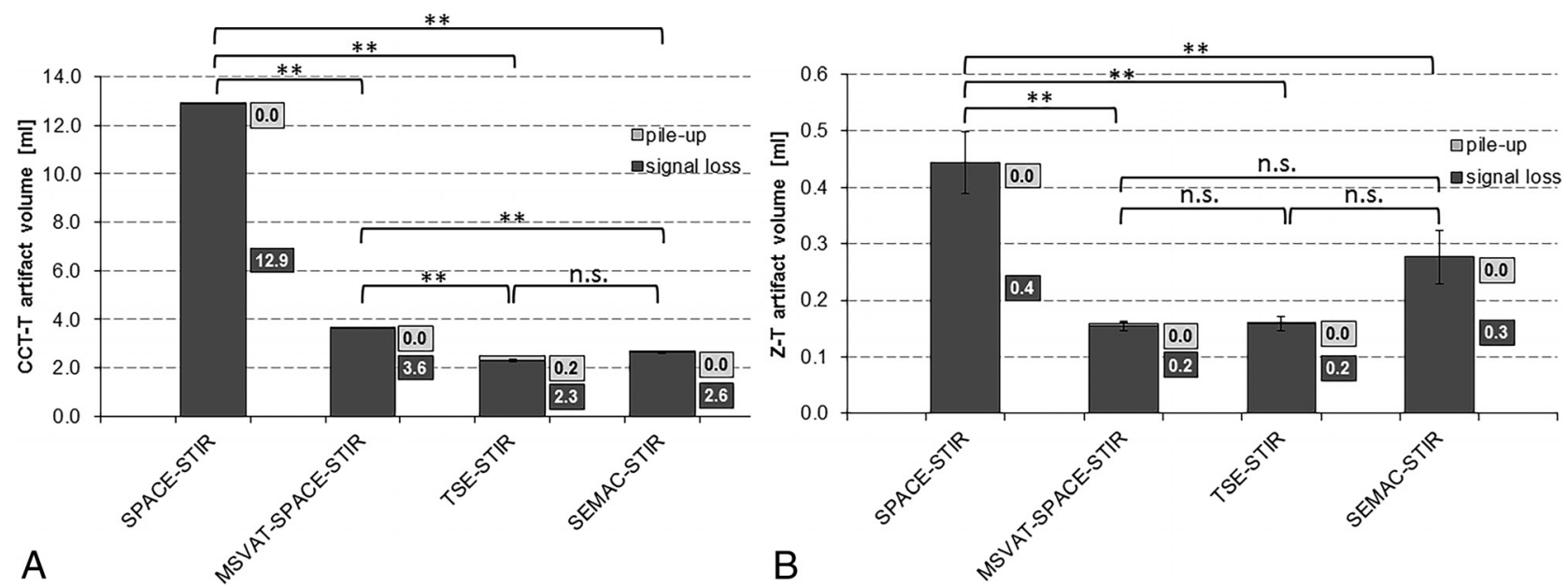

FIG 1. Artifact volumes (signal loss and pileup artifacts) of all sequences caused by the CCT-T $(A)$ and the Z-T (B) samples (double asterisks indicate $P \leq .001$; numbers next to the bars indicate the volume of pileup and signal loss artifacts separately in milliliters). n.s. indicates not significant.
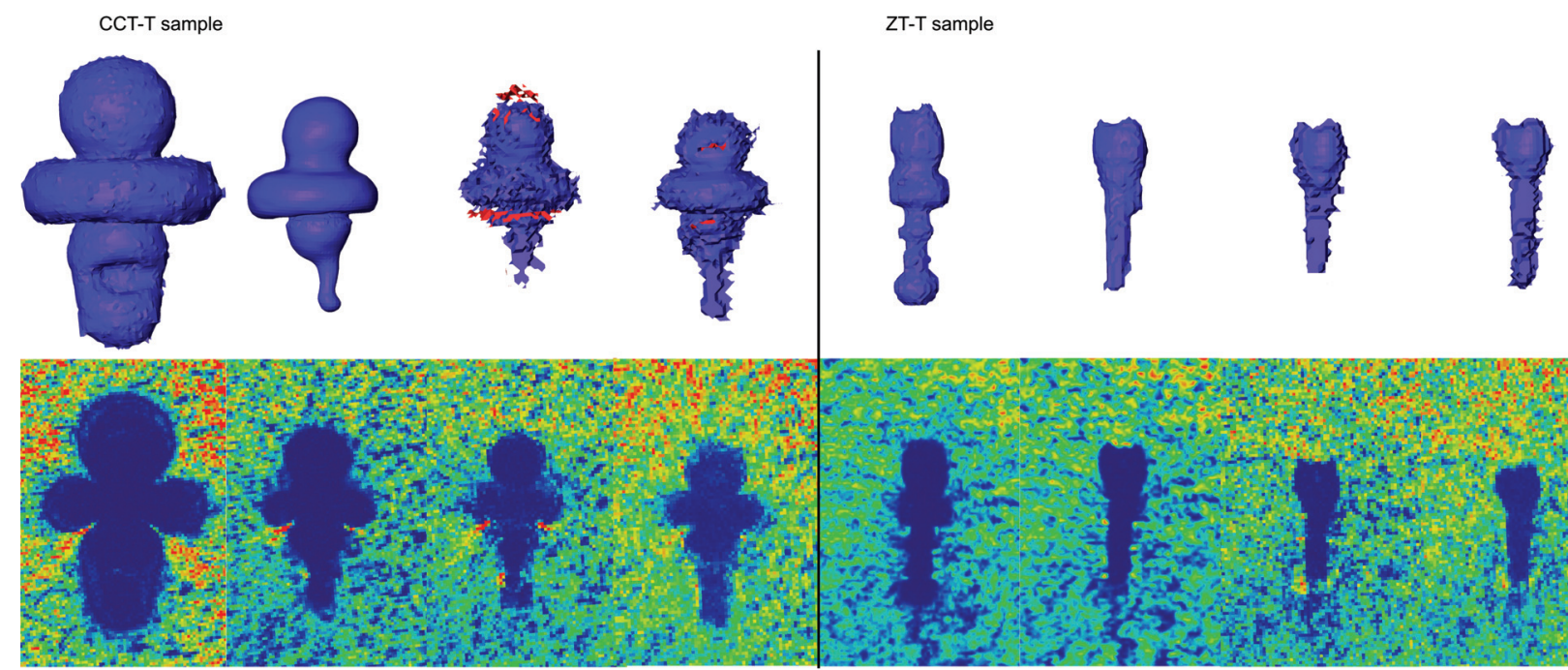

A SPACE-STIR

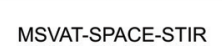

TSE-STIR

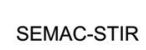

B SPACE-STR

FIG 2. 3D rendering of artifacts and source images (blue, signal loss artifacts; red, pileup artifacts) of the CCT-T ( $A$ ) samples and Z-T (B) samples for all evaluated sequences.

in the volunteers was improved in MSVAT-SPACE-STIR compared with standard SPACE-STIR (Fig 3). The smallest artifact volumes were observed for TSE-STIR and SEMAC-STIR followed by MSVAT-SPACE-STIR. In particular, the artifact volume of SEMAC-STIR was significantly smaller than the artifact volume of MSVAT-SPACE-STIR for the CCT-T sample $(P<$ $.001 ; 2.7$ versus $3.7 \mathrm{~mL})$ but not for the $\mathrm{Z}$ - $\mathrm{T}$ sample $(P=.974$; 0.3 versus $0.2 \mathrm{~mL}$ ).

The amount of pileup artifacts, as an indicator of slice distortions, was dependent on the applied sequence type. The proportion between pileup artifact volume and overall artifact volume was lower for SPACE-STIR/MSVAT-SPACE-STIR (mean, $0.007 \% \pm 0.0007 \% / 1.3 \% \pm 0.004 \%$ for the CCT sample) compared with TSE-STIR/SEMAC-STIR (mean, $7.8 \% \pm$ $0.07 \% / 0.99 \% \pm 0.04 \%$ ). SEMAC-STIR significantly reduced the amount of pileup artifacts on overall artifact size for both samples $(P<.001)$.

\section{Evaluation of Image Quality}

The nSNR of MSVAT-SPACE-STIR was higher than the nSNR of SPACE-STIR $(P<.001$, SNR increase of $22 \% \pm 4.5 \%$; Fig 4). No significant differences in nSNR were found between TSE-STIR and SEMAC-STIR. The nSNR of MSVAT-SPACESTIR was 4.8 times higher in comparison with SEMAC-STIR $(P<.001)$.

Interrater agreement for the assessment of image quality in porcine heads was good $(\kappa=0.67)$. Intrarater agreement was good for the first rater $(\kappa$-value $=0.77)$ and excellent for the second rater $(\kappa$-value $=0.86)$. No systematic differences in image quality were detected between SPACE-STIR and MSVAT-SPACE-STIR and TSE-STIR and SEMAC-STIR, respectively (Fig 5). Only the image quality of enamel/dentin was slightly better in TSE-STIR, SPACE-STIR, and MSVATSPACE-STIR compared with SEMAC-STIR $(P<.05, P<.001$, $P<.001$; Fig 5). 

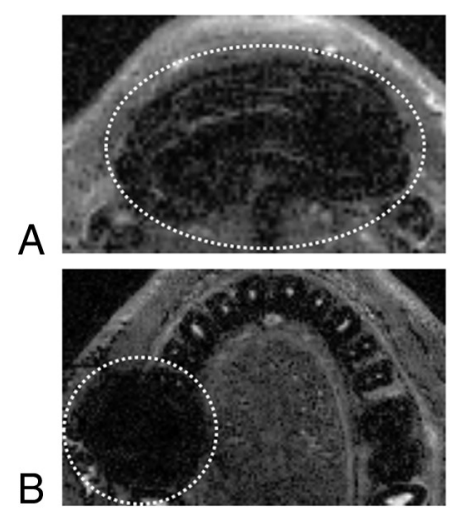

SPACE-STIR
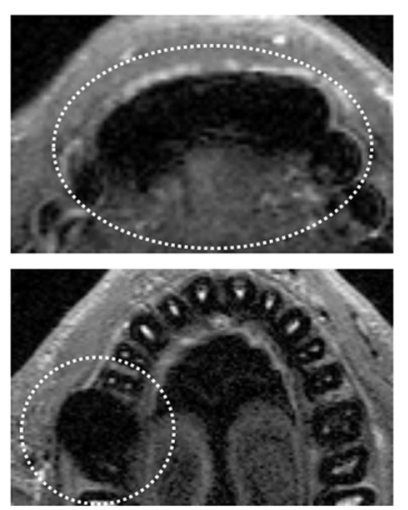

MSVAT-SPACE-STIR
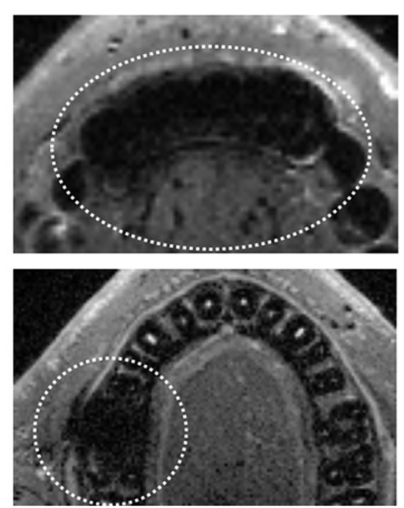

TSE-STIR
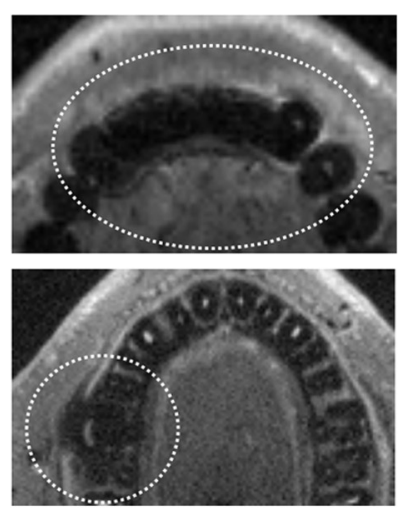

SEMAC-STIR

FIG 3. Comparison of all 4 STIR sequences in 2 volunteers with metallic dental materials. $A, A$ patient with artifacts caused by a retainer (signal-loss artifact within dashed lines). B, A volunteer with artifacts caused by an amalgam filling. Note the decrease of artifact sizes in MSVAT-SPACE-STIR images compared with SPACE-STIR images in both examples. Minor differences can be noted between TSE-STIR and SEMAC-STIR images, as well.

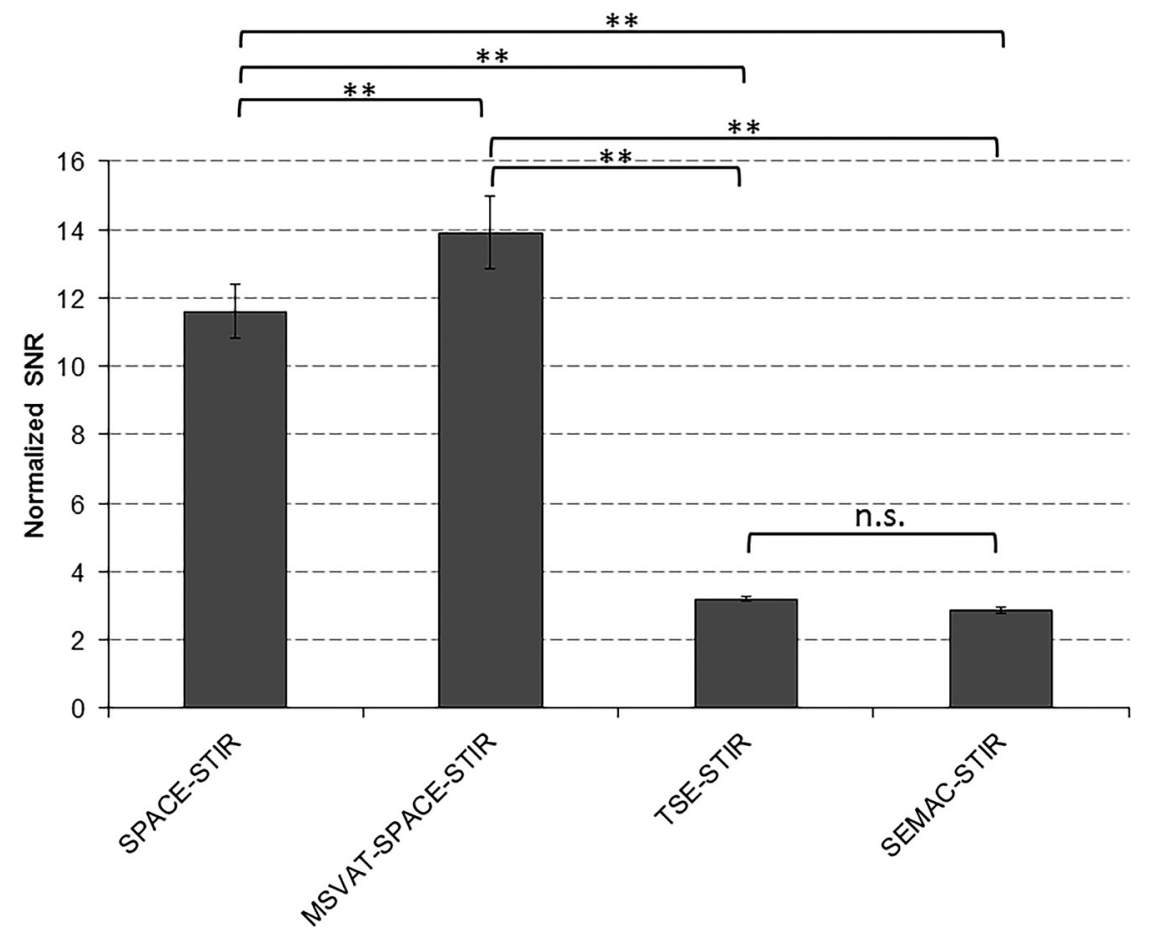

FIG 4. nSNR values of all used sequences. Double asterisks indicate $P \leq .001)$. n.s. indicates not significant.

\section{In Vivo Analysis}

Finally, all 4 STIR sequences were tested in 5 volunteers with metallic dental restorations or retainers (Fig 3). When we compared all sequences, the largest artifact areas were observed in SPACE-STIR $(P<.001)$. Furthermore, MSVAT-SPACE-STIR significantly reduced the artifact area in all volunteers compared with the SPACE-STIR sequence $(P<.001$; implant-supported crown, $35.7 \% \pm 1 \%$; retainer $1,25.2 \% \pm 0.3 \%$; retainer $2,54.3 \% \pm 1.2 \%$; metal abrasion, $36.2 \% \pm 3.1 \%$; amalgam filling, $60.4 \% \pm 5.8 \%$ ). The mean artifact reduction of MSVAT-SPACE-STIR was $42.3 \% \pm 14.5 \%$ compared with SPACE-STIR (Fig 3). In contrast, SEMAC-STIR significantly reduced the artifact area only for 1 volunteer with a retainer $(23.6 \% \pm 1.4 \% ; P<.001 ;$ Fig 3$)$ compared with TSE-STIR. As noted in the in vitro analysis, the smallest artifact areas were observed for TSE-STIR and SEMAC-STIR, followed by MSVATSPACE-STIR.

Because $>30$ minutes of acquisition time would have been needed for the 4 STIR sequences tested in vitro before, it was not possible to implement all sequences in clinical protocols. Because the visibility scores did not differ systematically and the results of artifact-reduction studies were dependent on the analyzed material, the nSNR and estimated size of artifacts in each patient were the decisive factors for sequence selection. Because none of the 5 randomly selected patients with head and neck pathologies presented with dental materials known to cause severe artifacts (eg, retainers), we chose to use MSVATSTIR instead of SEMAC-STIR because of higher resolution, higher nSNR, and isotropic voxel size (Fig 6). In clinical application, no or only minor motion artifacts were observed by both raters (mean score of motion artifacts of both raters and all subjects, $1.3 \pm 0.5$; range over all subjects, $1.2 \pm 0.4$ to $1.6 \pm 0.5)$.

\section{DISCUSSION}

In head and neck imaging, an increasing number of patients are presenting with metallic implants. ${ }^{6}$ This results in decreased image quality in the head and neck area and can even affect brain MR images. ${ }^{23}$ Sufficient image quality, however, is essential, for example, for staging oral cavity cancers, detecting injury of the inferior alveolar nerve, or detecting bone marrow enhancement and edema in osteomyelitis. Thus, artifact-reduction techniques are becoming increasingly important for the head and neck area. Prior studies have evaluated the benefit of artifact-reduction tech- 


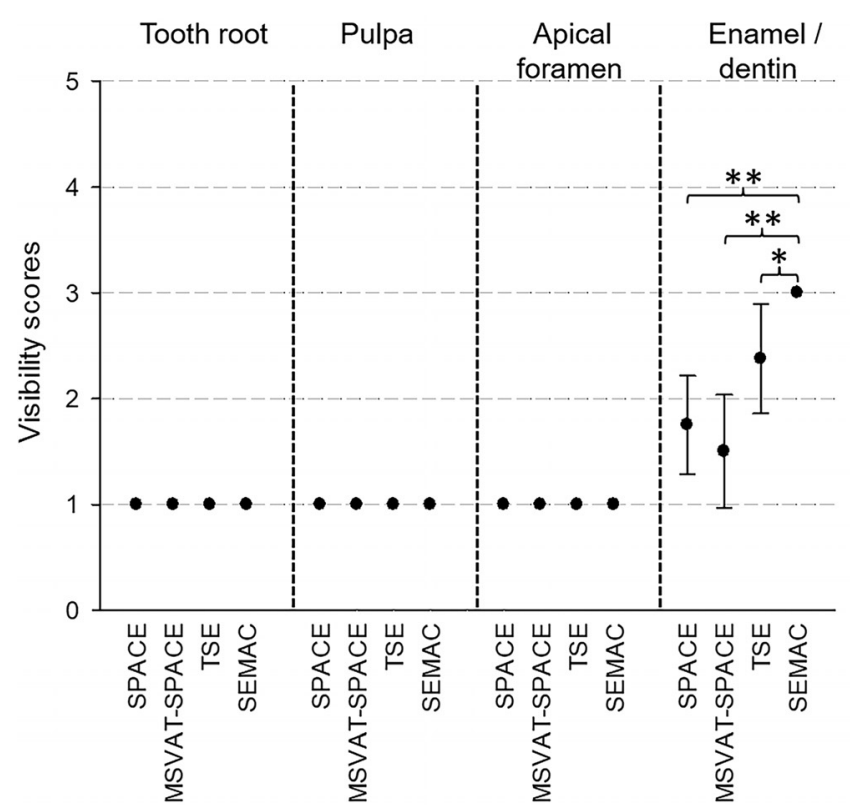

Sequences

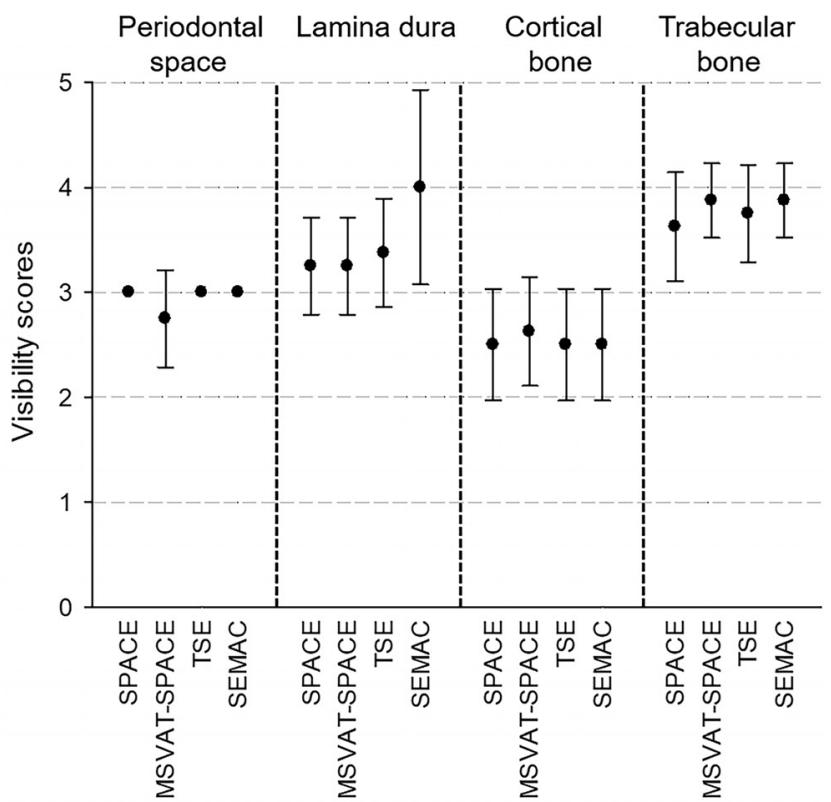

Sequences

FIG 5. Mean visibility scores of in vitro images of the 8 anatomic structures in all STIR sequences. The asterisk indicates $P \leq .05$; double asterisks, $P \leq .001$.

A
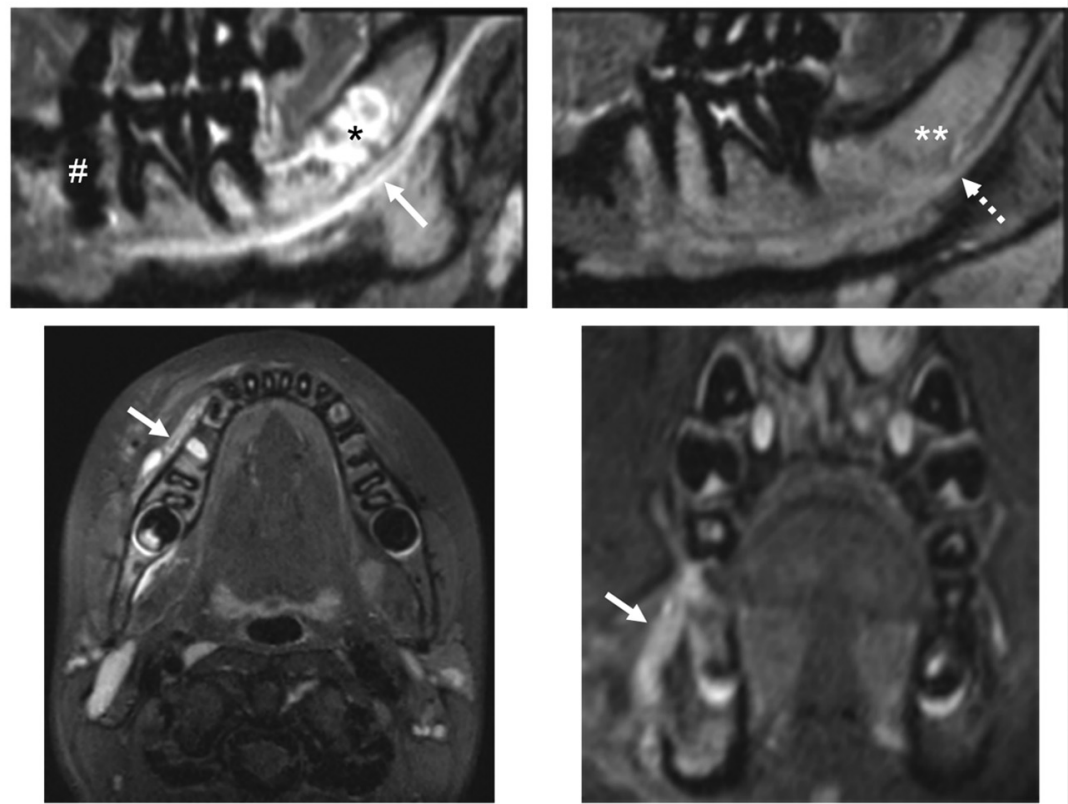

FIG 6. Two patients examined with MSVAT-SPACE-STIR. A, A 25-year-old woman with dysesthesia in the right mandible and chin after wisdom tooth extraction in the right mandible (asterisk indicates the extraction site). Increased signal intensity of the neurovascular bundle (white solid arrow) compared with the healthy side (white dashed arrow) in curved multiplane reconstructions of MSVAT-SPACE-STIR, suggesting nerve damage. Note the small amount of artifacts around the implant-supported crown (hash tag) and incomplete bone marrow conversion resulting in bright signal in STIR images on both sides (double asterisks). $B$, An 8-year-old child after drainage of an abscess in the right mandible with residual softtissue inflammation in the right lateral gingiva (white arrows in reformatted axial [left]) and coronal [right]) images).

niques for orthopedic and neurosurgical applications, but little attention has been paid to the head and neck area so far. Here, we demonstrate the advantages and disadvantages of MSVATSPACE-STIR and SEMAC-STIR in high-resolution head and neck imaging, with special regards to short acquisition times in vitro and in vivo.

The MSVAT-SPACE-STIR sequence revealed a significant artifact reduction compared with the standard SPACESTIR sequence in vivo and in vitro. No difference in artifact size was noted between MSVAT-SPACE-STIR and SEMACSTIR for materials with lower magneticsusceptibility difference compared with that of soft tissue. For materials with higher magnetic susceptibility, TSESTIR and SEMAC-STIR showed the smallest artifact volumes. A significant reduction of distortions was observed by SEMAC-STIR compared with TSESTIR. Combined artifact volume was not different between SEMAC-STIR and TSE-STIR in the in vitro analysis. In contrast, a small but statistically significant difference was observed in some volunteers, indicating a dependency of the results on material composition and material size.

Regarding MSVAT-SPACE-STIR, our results are consistent with the results of Ai et $\mathrm{al}^{7}$ who reported a reduction of artifact volume for MSVAT-SPACE in T1-weighted images at $1.5 \mathrm{~T}$ when imaging titanium screws. They observed a comparable degree of artifact reduction for titanium by comparing MSVAT-SPACE and conventional SPACE (up to 56\% mean reduction in comparison with $70.5 \%$ in our study). However, the authors did not evaluate 
the impact of 3T STIR imaging and, most important, dental restorations or implants, which are regularly encountered in clinical routine.

In contrast, a study by Zho et $\mathrm{al}^{24}$ reported an artifact reduction of $80 \%$ by non-STIR SEMAC using a dental crown made of nickel and chromium. The apparently differing results can be explained by the difference in the number of slice-encoding steps, resulting in differences in spectral coverage and artifact volume, respectively. Higher numbers of slice-encoding steps increase the spectral coverage and thereby reduce the size of artifacts but increase the acquisition time at the same time. Zho et al used 36 slice-encoding steps in an acquisition time of 29 minutes. Such long acquisition times are not applicable to in vivo head and neck imaging because different weightings as well as pre- and postcontrast images are typically used in clinical protocols. An in vivo study of Lee et $\mathrm{al}^{13}$ noted only a minor artifact reduction of $17.8 \%$ using SEMAC-STIR instead of TSE-STIR for spine imaging. Once again, this can be explained by less spectral coverage in terms of slice-encoding steps compared with Zho et al but still more than we used in our study: 11 (Lee et al) versus 36 (Zho et al) versus 4 in our study. With our sequence parameters, however, an increase of slice-encoding steps from 4 to 11 would still have resulted in an acquisition time of $>17$ minutes, which precludes clinical use.

Since image quality was not systematically different among all tested sequences, we conclude that artifact reduction does not come at the expense of image quality. However, in the case of SEMAC-STIR, artifact reduction resulted in 75\% increased acquisition time. Therefore, further acceleration techniques such as compressed sensing for the SEMAC sequence are desirable ${ }^{25}$ to facilitate the clinical applicability of this technique. In contrast, MSVAT-SPACE-STIR significantly reduced overall artifact volume and decreased acquisition time by $57 \%$ compared with SPACE-STIR. In addition, the nSNR of MSVAT-SPACE-STIR was nearly 5 times higher than the nSNR of SEMAC-STIR. Furthermore, MSVAT-SPACE-STIR, unlike SEMAC-STIR, allowed isotropic image acquisition and multiplane reconstructions. In vivo application of MSVAT-SPACE-STIR resulted in high-quality $3 \mathrm{D}$ datasets with decreased artifact size.

We acknowledge some limitations of our study. The T1 relaxation times of the phantom are not identical to those in living tissue. This feature may result in vivo in other artifact volumes in STIR sequences as in our in vitro results. Furthermore, our phantom design allowed only evaluation of slice distortions in direct proximity of the signal loss. Because additional slice distortions can be expected beyond the signal loss as well, we probably underestimated the amount of slice-distortion reduction by MSVAT-SPACE-STIR and SEMAC-STIR. Finally, because of the small number of patients included, further research is necessary to determine which sequence is best in a patient population.

\section{CONCLUSIONS}

For optimized fat suppression in the presence of metallic dental implants for head and neck imaging, MSVAT-SPACE-STIR reduced artifact volume and acquisition time compared with the standard SPACE-STIR sequence while maintaining image quality. In addition, MSVAT-SPACE-STIR allowed a much higher resolution than SEMAC-STIR and offered the possibility of 3D reconstructions. Within a clinically reasonable acquisition time, SEMAC-STIR reduced the amount of slice distortions, and, for some materials, artifact size as well compared with the optimized TSE-STIR sequence. Therefore, radiologists must decide between high-resolution 3D imaging (MSVAT-SPACE-STIR) and the smallest artifact size (SEMAC-STIR). SPACE-STIR is not recommended for head and neck MR imaging due to its vulnerability to susceptibility artifacts.

\section{ACKNOWLEDGMENTS}

The authors would like to thank Stefanie Sauer, $\mathrm{PhD}$, a pharmacist at the Department of Pharmacy, Heidelberg University Hospital, for her contribution to the MR imaging phantom. Furthermore, we would like to thank NORAS MRI Products, especially Daniel Gareis, MSc, and Celik Turgay, MSc, for providing the two 16-channel multipurpose coils used in the present study.

Disclosures: Tim Hilgenfeld—RELATED: Grant: Dietmar-Hopp Foundation.* Marcel Prager—RELATED: Grant: Dietmar Hopp Foundation*. Mathias Nittka—UNRELATED: Employment: Siemens, Germany. Peter Rammelsberg_UNRELATED: Board Membership: GrindCare (Sunstar), Comments: Scientific Board, financial compensation only for travelling expenses; Payment for Lectures Including Service on Speakers Bureaus: ZMK Update, Oemus Media. Martin Bendszus - UNRELATED: Board Membership: Data and Safety Monitoring Board for Vascular Dynamics, Guerbet, Boehringer Ingelheim; Consultancy: Codman Neuro, Roche AG, Guerbet, Boehringer Ingelheim, B. Braun Medical; Grants/Grants Pending: German Research Foundation, Dietmar Hopp Foundation, Novartis, Siemens, Guerbet, Stryker, Covidien*. Sabine Heiland-RELATED: Grant: Dietmar Hopp Foundation*; UNRELATED: Grants/ Grants Pending: German Research Foundation (SFB 1118)*. Alexander JuerchottRELATED: Grant: Dietmar Hopp Foundation*. *Money paid to the institution.

\section{REFERENCES}

1. Lissac M, Metrop D, Brugirard J, et al. Dental materials and magnetic resonance imaging. Invest Radiol 1991;26:40-45 CrossRef Medline

2. Schenck J. The role of magnetic susceptibility in magnetic resonance imaging: MRI magnetic compatibility of the first and second kinds. Med Phys1996;23:815-50 Medline

3. Lüdeke K, Röschmann P, Tischler R. Susceptibility artefacts in NMR imaging. Magn Reson Imaging 1985;3:329-43 CrossRef Medline

4. Camacho CR, Plewes DB, Henkelman RM. Nonsusceptibility artifacts due to metallic objects in MR imaging. J Magn Reson Imaging 1995;5:75-88 CrossRef Medline

5. Graf H, Steidle G, Martirosian P, et al. Metal artifacts caused by gradient switching. Magn Reson Med 2005;54:231-34 CrossRef Medline

6. Jordan RA, Micheelis W, Cholmakov-Bodechtel C, et al. Fifth German Oral Health Study. Köln: Deutscher Ärzte Verlag; 2016

7. Ai T, Padua A, Goerner F, et al. SEMAC-VAT and MSVAT-SPACE sequence strategies for metal artifact reduction in $1.5 \mathrm{~T}$ magnetic resonance imaging. Invest Radiol 2012;47:267-76 CrossRef Medline

8. Lee YH, Lim D, Kim E, et al. Usefulness of slice encoding for metal artifact correction (SEMAC) for reducing metallic artifacts in 3-T MRI. Magn Reson Imaging 2013;31:703-06 CrossRef Medline

9. Koch KM, Brau AC, Chen W, et al. Imaging near metal with a MAVRIC-SEMAC hybrid. Magn Reson Med 2011;65:71-82 CrossRef Medline

10. Cho ZH, Kim DJ, Kim YK. Total inhomogeneity correction including chemical shifts and susceptibility by view angle tilting. Med Phys 1988;15:7-11 CrossRef Medline

11. Lu W, Pauly KB, Gold GE, et al. SEMAC: slice encoding for metal artifact correction in MRI. Magn Reson Med 2009;62:66-76 CrossRef Medline

12. Koch KM, Lorbiecki JE, Hinks RS, et al. A multispectral three-dimensional acquisition technique for imaging near metal implants. Magn Reson Med 2009;61:381-90 CrossRef Medline 
13. Lee YH, Hahn S, Kim E, et al. Fat-suppressed MR imaging of the spine for metal artifact reduction at 3T: comparison of STIR and slice encoding for metal artifact correction fat-suppressed T2weighted images. Magn Reson Med Sci 2016;15:371-78 CrossRef Medline

14. Sutter R, Ulbrich EJ, Jellus V, et al. Reduction of metal artifacts in patients with total hip arthroplasty with slice-encoding metal artifact correction and view-angle tilting MR imaging. Radiology 2012; 265:204-14 CrossRef Medline

15. Fleckenstein JL, Archer BT, Barker BA, et al. Fast short-tau inversion-recovery MR imaging. Radiology 1991;179:499-504 CrossRef Medline

16. Cox B, Zuniga JR, Panchal N, et al. Magnetic resonance neurography in the management of peripheral trigeminal neuropathy: experience in a tertiary care centre. Eur Radiol 2016;26:3392-400 Medline

17. Koch KM, Hargreaves BA, Pauly KB, et al. Magnetic resonance imaging near metal implants. J Magn Reson Imaging 2010;32:773-87 CrossRef Medline

18. Li G, Nittka M, Paul D, et al. MSVAT-SPACE for fast metal implants imaging. Proceedings of the ISMRM 2011;19:3171

19. Hilgenfeld T, Prager M, Schwindling FS, et al. Artefacts of implantsupported single crowns: impact of material composition on arte- fact volume on dental MRI. Eur J Oral Implantol 2016;9:301-08 Medline

20. Dietrich O, Raya JG, Reeder SB, et al. Measurement of signal-tonoise ratios in MR images: influence of multichannel coils, parallel imaging, and reconstruction filters. J Magn Reson Imaging 2007;26: 375-85 CrossRef Medline

21. Friedman L, Glover GH. Report on a multicenter fMRI quality assurance protocol. J Magn Reson Imaging 2006;23:827-39 CrossRef Medline

22. Landis J, Koch GG. An application of hierarchical kappa-type statistics in the assessment of majority agreement among multiple observers. Biometrics 1977;33:363-74 CrossRef Medline

23. Costa ALF, Appenzeller S, Yasuda C-L, et al. Artifacts in brain magnetic resonance imaging due to metallic dental objects. Med Oral Patol Oral Cir Bucal 2009;14:82 Medline

24. Zho SY, Kim MO, Lee KW, et al. Artifact reduction from metallic dental materials in T1-weighted spin-echo imaging at 3.0 Tesla. $J$ Magn Reson Imaging 2013;37:471-78 CrossRef Medline

25. Fritz J, Ahlawat S, Demehri S, et al. Compressed sensing SEMAC: 8-fold accelerated high resolution metal artifact reduction MRI of cobalt-chromium knee arthroplasty implants. Invest Radiol 2016; 51:666-76 CrossRef Medline 